\title{
Characteristics of COVID-19 During the Onset Stage and Considerations for Disease Control
}

\author{
Lili Wang* \\ Department of Medicine, Division of Infectious Diseases, Icahn School of Medicine at Mount Sinai, New York, NY, 10029, USA
}

\begin{abstract}
COVID-19 outbreak in December 2019 has evolved into a world level pandemic. To cope with an emerging pathogen like SARS-CoV-2, there is no vaccine or specific drug available to treat the disease. However, get to know the characters of the onset stage of the new disease may help us to control the spread of COVID-19. This short article aims to introduce COVID-19 briefly, getting you to know the clinical character, the treatment, public procedurals and researches on COVID-19 quickly but comprehensively.
\end{abstract}

The first pneumonia case of unknown origins was identified in Wuhan, China in early December $2019 .{ }^{1}$ The symptoms of it reminded people of severe acute respiratory syndrome coronavirus (SARS-CoV), which had shocked China 17 years ago in 2003. High-throughput sequencing confirmed the new virus is the seventh member of enveloped RNA coronavirus (subgenus sarbecovirus, Orthocoronavirinae subfamily). Currently, the new virus was named as severe acute respiratory syndrome coronavirus 2 , SARS-CoV-2), which was also called 2019 novel coronavirus (2019-nCoV) earlier. And the disease caused by it was named coronavirus disease in 2019 (COVID-19). ${ }^{2,3}$ Until now, COVID-19 outbreaks emerged in Wuhan have caused more than 80,000 confirmed infections; including more than 2,800 deaths (December 1st, 2019-Feb 29th 2020, 3 months). On January 30, 2020, the International Health Regulations Emergency Committee of the World Health Organization declared the outbreak a "public health emergency of international concern". To cope with an emerging pathogen with serious outcomes, the first thing to do is get to know the enemy. Scientists and doctors in the frontier responded quickly to this emergency and provided valuable epidemiological and clinical data sharing with the rest of the world before its further spread. Knowing what happened during the onset of the diseases, and what test method, diagnosis and treatment was performed is important for us to assess the procedurals, and further evaluate and control the disease.

Keywords: COVID-19; SARS-CoV-2.

Abbreviations: SARS-CoV, severe acute respiratory syndrome coronavirus; 2019nCoV, 2019 novel coronavirus; COVID-19, coronavirus disease in 2019; SARS$\mathrm{CoV}-2$, severe acute respiratory syndrome coronavirus 2; ARDS, acute respiratory distress syndrome.

Received: February 14, 2020; Revised: February 29, 2020; Accepted: March 02, 2020

${ }^{*}$ Correspondence to: Lili Wang, Department of Medicine, Division of Infectious Diseases, Icahn School of Medicine at Mount Sinai, New York, NY, 10029, USA. Tel: +1212-241-2503, E-mail: lili.wang@mssm.edu

How to cite this article: Wang L. Characteristics of COVID-19 During the Onset Stage and Considerations for Disease Control. Exploratory Research and Hypothesis in Medicine 2020;5(1):8-10. doi: 10.14218/ERHM.2020.00005.
Epidemiological characteristics

According to the first published descriptive study based on 99 patients from Jinyintan Hospital in Wuhan, ${ }^{4} 49 \%$ of the $2019-\mathrm{nCoV}$ infected patients had a history of exposure to Huanan Seafood Wholesale Market. 2019-nCoV infection was of clustering onset, was more likely to affect older men with comorbidities, and could result in severe and even fatal respiratory diseases such as acute respiratory distress syndrome (ARDS). A later study published on Jan 29th with a sample size of 425 cases indicates the majority of cases (55\%) with onset before Jan $1^{\text {st }}, 2020$ were linked to the Huanan Seafood wholesale Market, while after that date only $8.6 \%$. The mean incubation period was 5.2 days $(95 \%$ confidence interval, 4.1 to 7.0$)$ and the basic reproductive number was estimated to be 2.2 (95\% confidence interval, 1.4 to 3.9$).{ }^{5}$ These data indicate human-to-human transmission has occurred among close contacts since the middle of December 2019.

\section{Clinical characteristics}

Early reports from Huang et al. on Jan 24, 2020, provided clinical characteristics of 41 patients confirmed with 2019-nCoV infection in Wuhan Jinyintan Hospital. These data indicated that patients' clinical manifestations included fever, nonproductive cough, dyspnea, myalgia, fatigue, Lymphopenia, and radiographic evidence of pneumonia. Organ dysfunction (e.g., shock, acute respiratory distress syndrome (ARDS), acute cardiac injury, and acute kidney injury) and death can occur in severe cases. ${ }^{1} 138$ patients confirmed with 2019-nCoV infection from Zhongnan Hospital of Wuhan University showed similar clinical characteristics based on the report on Feb 7 from Wang et al. ${ }^{6}$ In this report, one thing to notice is that infection for affected health professionals and hospitalized patients take $29 \%$ and $12.3 \%$ respectively. This suggests among the 138 patients, $41.3 \%$ got infected inside the hospital. Case reports confirmed human-to-human transmission of 2019-nCoV. ${ }^{7,8}$ On the national scale, data of 1,099 patients from 552 hospitals in 31 provinces were assessed. ${ }^{9}$ Based on this study preprinted online on 
Table 1. The summary of the most commonly seen symptom from studies

\begin{tabular}{|c|c|c|c|c|c|c|c|}
\hline & Sample size & Median age & Fever & Fatigue & Dry cough & Dyspnea & Lymphopenia \\
\hline Jinyintan Hospital ${ }^{1}$ & 41 & 49 & $98 \%$ & $44 \%$ & $31 \%$ & $22 \%$ & $63 \%$ \\
\hline Zhongnan Hospital ${ }^{6}$ & 138 & 56 & $98.6 \%$ & $69.6 \%$ & $59.4 \%$ & $31.2 \%$ & $70.3 \%$ \\
\hline National ${ }^{9}$ & 1,099 & 47 & $87.9 \%$ & $38.1 \%$ & $67.7 \%$ & $18.6 \%$ & $82.1 \%$ \\
\hline
\end{tabular}

Feb 6, 31.30\% of the patients had been to Wuhan and $71.80 \%$ had contact people from Wuhan. This paper also indicated the median incubation period was 3.0 days (range, 0 to 24.0 days). The most commonly seen symptom from these studies was summarized in Table 1.1,69 According to the 1,099 people study, $18.6 \%$ of the patients were severe cases (based on American Thoracic Society guideline), and $5 \%$ of them were treated in ICU. The death rate is around $2 \%$ based on these reports.

\section{Treatment}

As the SARS-CoV-2 is a novel coronavirus that just been identified, no specific drug has been found to treat COVID-19. Based on the experiences gained on SARS-CoV infection, the early patients were treated with antibiotics and oseltamivir. Corticosteroid therapy was given as a combined regimen if severe pneumonia was diagnosed. Oxygen support was administered to patients according to the severity of hypoxemia. ${ }^{1,6,9}$ In vitro studies on antiSARS-CoV-2 drugs have shown high efficacy of remdesivir. ${ }^{10}$ The first confirmed COVID patient in the U.S. was cured by remdesivir. ${ }^{11}$ At present at least two phase-3 clinical trials on remdesivir were carried out from early February 2020. With more patients recovered from COVID-19 and released from the hospital, donated blood plasma from recovered patients with antibodies against SARS-CoV-2 is also used to treat severe cases. Other remedies are also performed to reduce the damage of vital organs by cytokine storm induced by the immune response. ${ }^{12}$

\section{Comments}

A confirmed case with COVID-19 was defined as a positive result to real-time reverse-transcriptase polymerase-chain-reaction (RTPCR) assay or high-throughput sequencing for nasal and pharyngeal swab specimens. ${ }^{1}$ However, when an individual was infected but with low viral load, the test result could be negative due to the relatively high false-negative rate of the PCR based tests. This might potentially lead to the spread of SARS-CoV-2. There are cases with negative results in the early days of COVID-19 when the symptom is not severe but later confirmed as positive SARSCoV-2 infection. ${ }^{13}$ Self-quarantine for 14 days when has suspicious syndrome is a safe strategy. SARS-CoV-2 spread rapidly through human-to-human routes, accumulating tens of thousands of patients within a single month in Wuhan. The huge number of patients that emerged during such a short time saturated the medical resources. How to properly isolate and treat patients has become a great challenge to the public health system.

\section{Perspectives}

Although several promising trials are carried out in China and America, at present no specific drugs have been confirmed to cure
COVID-19. The vaccines are still under development. A cryo-EM study on 2019-nCoV spike protein revealed the prefusion conformation of spike glycoprotein of 2019-nCoV and found it can bind to the receptor ACE2 with higher affinity than its SARS-CoV counterpart. ${ }^{14}$ As the spike glycoprotein is a key target for vaccines, therapeutic antibodies and diagnostics, the discoveries in the details of spike glycoprotein structure will contribute to the drug and vaccine development. Drugs against coronavirus RNAdependent RNA polymerase is also under clinical trial. Due to the high infectivity of SARS-CoV-2, the optimal strategies against spreading remains to be early detection and early quarantine. The early detection relies on a quick and accurate detection method, which is also under clinical test. Until Feb 29, 2020, the number of newly diagnosed people in China has declined for over a week, which is a good sign of COVID-19 under control. However, new patients number confirmed in other countries is increasing. ${ }^{15}$ Rigid control by the government would be crucial under these public emergency circumstances. In this article, I hope all the endeavors we made will shorten the course of this modern plague, with the least spreading to the other part of the world.

\section{Acknowledgments}

I thank the colleges from Icahn School of Medicine for meaningful discussion.

\section{Funding}

This article is not fund-supported.

\section{Conflict of interest}

The author has no conflict of interest related to this publication.

\section{References}

[1] Huang C, Wang Y, Li X, Ren L, Zhao J, Hu Y, et al. Clinical features of patients infected with 2019 novel coronavirus in Wuhan, China. Lancet 2020;395(10223):497-506. doi:10.1016/S0140-6736(20)30183-5.

[2] Lu R, Zhao X, Li J, Niu P, Yang B, Wu H, et al. Genomic characterisation and epidemiology of 2019 novel coronavirus: implications for virus origins and receptor binding. Lancet 2020;395(10224):565-574. doi:10.1016/S0140-6736(20)30251-8.

[3] Zhu N, Zhang D, Wang W, Li X, Yang B, Song J, et al. A Novel Coronavirus from Patients with Pneumonia in China, 2019. N Engl J Med 2020;382:727-733. doi:10.1056/NEJMoa2001017.

[4] Chen N, Zhou M, Dong X, Qu J, Gong F, Han Y, et al. Epidemiological and clinical characteristics of 99 cases of 2019 novel coronavirus pneumonia in Wuhan, China: a descriptive study. Lancet 2020;395(10223):507-513. doi:10.1016/S0140-6736(20)30211-7.

[5] Li Q, Guan X, Wu P, Wang X, Zhou L, Tong Y, et al. Early Transmission 
Dynamics in Wuhan, China, of Novel Coronavirus-Infected Pneumonia. N Engl J Med 2020. doi:10.1056/NEJMoa2001316.

[6] Wang D, Hu B, Hu C, Zhu F, Liu X, Zhang J, et al. Clinical Characteristics of 138 Hospitalized Patients With 2019 Novel CoronavirusInfected Pneumonia in Wuhan, China. JAMA 2020. doi:10.1001/ jama.2020.1585.

[7] Chan JF, Yuan S, Kok KH, To KK, Chu H, Yang J, et al. A familial cluster of pneumonia associated with the 2019 novel coronavirus indicating person-to-person transmission: a study of a family cluster. Lancet 2020;395(10223):514-523. doi:10.1016/S0140-6736(20)30154-9.

[8] Phan LT, Nguyen TV, Luong QC, Nguyen TV, Nguyen HT, Le HQ, et al. Importation and Human-to-Human Transmission of a Novel Coronavirus in Vietnam. N Engl J Med 2020;382:872-874. doi:10.1056/ NEJMc2001272.

[9] Guan WJ, Ni ZY, Hu Y, Liang WH, Ou CQ, He JX, et al. Clinical Characteristics of Coronavirus Disease 2019 in China. N Engl J Med 2020. doi:10.1056/NEJMoa2002032.

[10] Wang M, Cao R, Zhang L, Yang X, Liu J, Xu M, et al. Remdesivir and chloroquine effectively inhibit the recently emerged novel corona- virus (2019-nCoV) in vitro. Cell Res 2020;30:269-271. doi:10.1038/ s41422-020-0282-0.

[11] Holshue ML, DeBolt C, Lindquist S, Lofy KH, Wiesman J, Bruce $\mathrm{H}$, et al. First Case of 2019 Novel Coronavirus in the United States. N Engl J Med 2020;382:929-936. doi:10.1056/NEJMoa2001191.

[12] Xu Z, Shi L, Wang Y, Zhang J, Huang L, Zhang C, et al. Pathological findings of COVID-19 associated with acute respiratory distress syndrome. Lancet Respir Med 2020. doi:10.1016/S2213-2600(20)30076$X$.

[13] Zhou Y, Yang L, Han M, Huang M, Sun X, Zhen W, et al. Clinical Reports on Early Diagnosis of Novel Coronavirus (2019-nCoV) Pneumonia in Stealth Infected Patients. Preprints 2020:2020020156. doi:10.20944/ preprints202002.0156.v1.

[14] Wrapp D, Wang N, Corbett KS, Goldsmith JA, Hsieh CL, Abiona O, et al. Cryo-EM structure of the 2019-nCoV spike in the prefusion conformation. Science 2020. doi:10.1126/science.abb2507.

[15] Hodcroft EB. Preliminary case report on the SARS-CoV-2 cluster in the UK, France, and Spain. Swiss Med Wkly 2020;150:w20212. doi:10.4414/smw.2020.20212. 